\title{
Non-steroidal anti-inflammatory drug-induced enteropathy
}

\author{
Sung Jae Shin, Choong-Kyun Noh, Sun Gyo Lim, Kee Myung Lee, Kwang Jae Lee \\ Department of Gastroenterology, Ajou University School of Medicine, Suwon, Korea
}

Non-steroidal anti-inflammatory drugs (NSAIDs) are well known to be associated with serious upper gastrointestinal complications, such as peptic ulcer, bleeding, perforation, and obstruction. Recently, attention has been mainly focused on the small bowel injuries caused by NSAIDs, and new endoscopic techniques such as capsule endoscopy and double balloon endoscopy can help in detecting such injuries. This article reviewed the epidemiology, pathogenesis, clinical manifestations, diagnosis, and treatment of small bowel injuries caused by NSAIDs. Small bowel injures by NSAIDs might occur with a similar frequency and extent as those observed in the upper gastrointestinal tract. The pathogenesis of NSAID-induced enteropathy is complex and not clearly understood. The various lesions observed in the small bowel, including petechiae, reddened folds, loss of villi, erosions, and ulcers can be detected by capsule endoscopy. A drug that could prevent or treat NSAID-induced enteropathy has not yet been developed. Therefore, further investigations should be performed to elucidate the pathogenesis of such enteropathy and develop suitable preventive and treatment strategies. (Intest Res 2017;15:446-455)

Key Words: Anti-inflammatory agents, non-steroidal; Small intestine injury; Capsule endoscopy

\section{INTRODUCTION}

Non-steroidal anti-inflammatory drugs (NSAIDs) are some of the most commonly prescribed drugs in the world. As a result of their anti-inflammatory, analgesic, and antiplatelet effects, NSAIDs are used in clinical practice for treatment and prevention of rheumatoid arthritis, osteoarthritis, collagen disease, and ischemic cardiovascular or cerebrovascular disease. However, NSAIDs are well known to increase the risk of serious gastroduodenal complications, such as peptic ulcer, bleeding, and perforations. ${ }^{1,2}$

Until recently, many studies regarding NSAID-induced gastrointestinal (GI) injuries were focused on the stomach and duodenum because of easy accessibility via upper en-

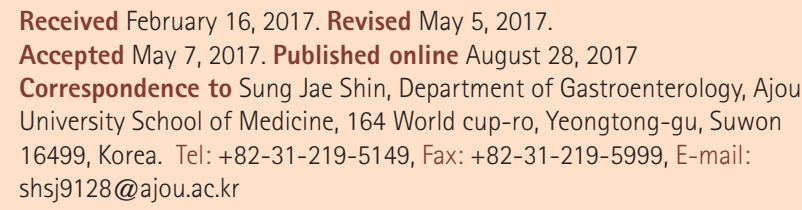

Financial support: None. Conflict of interest: None. doscopy. However, recent advances in diagnostic devices used to look at the small intestine, such as capsule endoscopy (CE) and double balloon endoscopy (DBE), enabled direct visualization of the small bowel, and revealed the small bowel injuries induced by NSAIDs. ${ }^{3-7}$ Graham et al. ${ }^{3}$ reported that small bowel damage was found in $71 \%$ of chronic NSAID users. However, unlike the NSAID-induced gastropathy, the symptoms of NSAID-induced enteropathy are non-specific and the pathogenesis is poorly understood. Additionally, there is no proven effective treatment or prevention for NSAID-induced enteropathy.

Therefore, studies to investigate the mechanism of NSAIDinduced enteropathy and treatment modalities for small bowel damage should be performed in the future. In this paper, we intend to describe the current status of small bowel injuries caused by NSAIDs.

\section{EPIDEMIOLOGY}

Over the last 10 years, there has been a progressive trend in overall GI complications, such as bleeding and perfora-

\footnotetext{
(c) Copyright 2017. Korean Association for the Study of Intestinal Diseases. All rights reserved.

This is an Open Access article distributed under the terms of the Creative Commons Attribution Non-Commercial License (http://creativecommons.org/licenses/by-nc/4.0)

which permits unrestricted non-commercial use, distribution, and reproduction in any medium, provided the original work is properly cited.
} 
tion, with a decrease in upper GI complications and an increase in lower GI complications, which include the jejunum, ileum, and large bowel. Thus, the ratio of upper to lower complications has changed from 4.1 in 1996 to 1.4 in $2005 .^{8}$

Among NSAID users, a 15\% prevalence of gastric ulcer and a $10 \%$ prevalence of duodenal ulcer was found in an endoscopic study. ${ }^{9}$ There are several reasons that make it difficult to recognize the prevalence of NSAID-induced enteropathy. First, observing small bowel injuries, induced by NSAIDs, is more difficult than those in the upper GI tract. Second, there is a poor correlation between NSAID-induced small intestinal damage and clinical symptoms. Most symptoms caused by NSAID-induced enteropathy are subclinical and nonspecific. ${ }^{10,11}$ Therefore, until the introduction of CE and DBE, the importance of NSAID-induced enteropathy had been underestimated, compared with NSAID-induced gastropathy.

Recent studies suggested that the damage to the small bowel may occur as frequently and be as severe as upper GI complications. ${ }^{10,11}$ Allison et al. ${ }^{12}$ reported the prevalence of small intestinal injuries (ulcerations) in NSAID users and non-users in post mortem patients. Small intestinal ulceration was found in $8.4 \%$ of the NSAID users and $0.6 \%$ of the non-users. In a study looking at results of capsule enteroscopies, Maiden et al. ${ }^{4}$ found that small bowel injuries were observed in $68 \%$ of healthy volunteers taking diclofenac plus omeprazole for 2 weeks. In another study with 28 rheumatoid arthritis patients, small bowel damage was detected in 13 of 16 patients (81.3\%) who used NSAIDs and in 4 of 12 patients (33.3\%) who did not. ${ }^{5}$ In the study using DBE, the NSAID enteropathy occurred in $51 \%$ of the patients taking NSAIDs. ${ }^{13}$ Aspirin seemed to be less harmful to the small bowel in comparison with other NSAIDs. ${ }^{14-16}$ However, even low-dose aspirin can cause bowel damage with shortterm administration. Endo et al. ${ }^{14}$ reported that small bowel injuries occurred more frequently in healthy volunteers to whom low-dose enteric-coated aspirin was administered for 14 days, than in the group not given any drug ( $80 \%$ vs. $20 \%$, $P=0.023$ ). Therefore, considering these results, it is necessary to be aware of the adverse effect of NSAIDs not only on the upper GI tract but also the lower GI tract.

\section{PATHOGENESIS}

Unlike that of the upper GI tract, the pathogenesis of small bowel injuries caused by NSAIDs was not clearly elucidated in the past because various multiple complicating factors were thought to influence the development of NSAIDinduced enteropathy.

Bjarnason et al. ${ }^{17}$ suggested a 3-hit hypothesis. First, the phospholipid in the cell membrane on the mucosal surface is directly damaged by NSAIDs and subsequently, injury of the mitochondria in the cell occurs. Second, mitochondrial damage induces the decrease of energy synthesis, resulting in calcium efflux and generation of free radicals. Then, disruption of intercellular junctions and increase of mucosal permeability develop. Third, the intraluminal contents, such as bile acid, proteolytic enzymes, intestinal bacteria, and toxins, can invade the cell through the weakened mucosal barrier, and inflammation develops (Fig. 1). ${ }^{18}$

PGs have an important role in GI blood flow and mucus production. The depletion of PGs by NSAIDs induces small bowel damage. ${ }^{19-22}$ There are 2 type of cyclooxygenase (COX). COX-1 induces PG synthesis and has an important role in maintaining the homeostasis of intestinal mucosa. Previously, only COX-1 inhibition was thought to be associated with mucosal injuries. However, in a recent animal model study, small intestinal damage developed only when both COX-1 and COX-2 were inhibited. ${ }^{23}$ This indicates that COX-2-derived PGs also play an important role in maintenance of the integrity of the tissue, repairing of mucosal injury, and resolution of inflammation. One study reported that the small bowel injuries by non-selective NSAIDs and selective COX-2 inhibitors were not significantly different in patients on long-term NSAIDs $(62 \%$ with conventional NSAIDs vs. 50\% with selective COX-2 inhibitors, $P$-value not significant) ${ }^{6}$

Enterohepatic circulation of NSAIDs also has an important role in the pathogenesis of NSAID-induced enteropathy. ${ }^{24-26}$ The topical adverse effect of aspirin was thought to be localized in the gastroduodenum because of rapid absorption in the stomach and duodenum and the lack of enterohepatic recirculation. To reduce gastric mucosal injuries caused by the topical irritant effect of aspirin, enteric-coated aspirin has been developed. ${ }^{27-30}$ Enteric-coated aspirin dissolves mainly in the small bowel, rather than the stomach or duodenum, and enters the enterohepatic circulation, which damages mostly the distal part of the small intestine. When NSAIDs or aspirin did not re-circulate enterohepatically, NSAID-induced small bowel injuries could not develop. ${ }^{31,32}$ One study reported that small bowel ulcers occurred more frequently in patients taking enteric-coated aspirin (56.3\%) than noncoated aspirin $(16.7 \%){ }^{14}$

Intestinal bacteria are important in the pathogenesis of NSAID-induced enteropathy. Germ-free rats and mice 


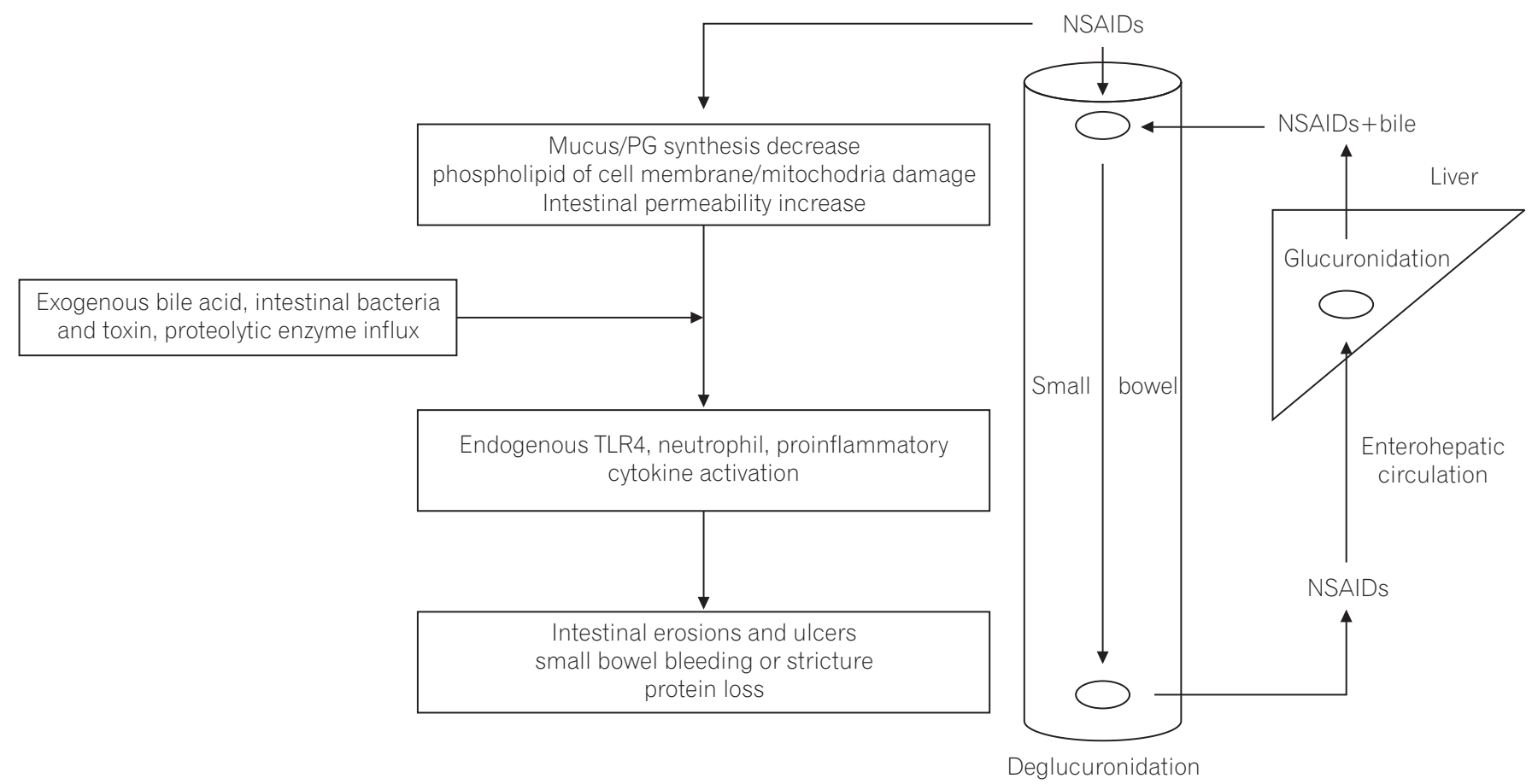

Fig. 1. Putative pathophysiology of NSAID-induced enteropathy. NSAIDs decrease PG synthesis, resulting in the reduction of mucus and blood flow of the intestinal mucosa and directly damage the small bowel epithelium. These injuries cause increased intestinal permeability of bile acid, bacteria, proteolytic enzymes, and toxins. Therefore, neutrophils and many inflammatory mediators are activated and various clinical manifestations, such as erosion, ulceration, bleeding, and protein loss develop. Additionally, enterohepatic circulation of NSAIDs and bile augments the damage to the small bowel. TLR4, Toll-like receptor 4. Adapted from Wallace JL. Br J Pharmacol 2012;165:67-74. ${ }^{18}$

had little or no intestinal injuries when given NSAIDs, but when the bowel was colonized by gram-negative bacteria, the small intestine was susceptible to injuries caused by NSAIDs. ${ }^{19,33}$ In addition, some antibiotics, which work against gram-negative bacteria, were effective in reducing NSAID-induced enteropathy. ${ }^{33-35}$ The lipopolysaccharide of gram-negative bacteria activated the Toll-like receptor 4 , which stimulated an inflammatory response, such as cytokine activation and tumor necrosis factor $\alpha$, triggering nitric oxide (NO) derived from inducible NO synthase and neutrophil activation, resulting in small bowel mucosal injuries. ${ }^{34-39}$ The NSAIDs, acryl glucuronides, which are excreted from the hepatocytes into the bile duct, are deconjugated by an intestinal bacterial enzyme, $\beta$-glucuronidase. Subsequently, NSAIDs could be transported across the epithelium and enter the enterohepatic circulation. The inhibition of $\beta$-glucuronidase in an animal model reduced the diclofenacinduced small bowel injuries. ${ }^{40}$ The low acidity of the gastric environment suppresses bacteria; therefore, chronic acid suppression by proton pump inhibitors (PPIs) can induce bacterial overgrowth and exacerbate the NSAID-induced enteropathy through dysbiosis. ${ }^{41-44}$ Similarly, probiotics could decrease the severity of NSAID-induced enteropathy. ${ }^{45}$

\section{CLINICAL SYMPTOMS AND SIGNS}

The signs and symptoms of NSAID-induced enteropathy are usually nonspecific. They have various manifestations, such as iron-deficiency anemia, protein loss, indigestion, constipation, diarrhea, and abdominal pain. ${ }^{10,11}$ Serious clinical complications, i.e., bleeding, obstruction, and perforation, are infrequent, but can be life threatening.

NSAID-induced enteropathy is associated with occult or overt GI bleeding, resulting in iron-deficiency anemia. In CE, ulcerations and erosions are found commonly in patients taking NSAIDs. ${ }^{4-7}$ Kameda et al ${ }^{46}$ reported that NSAIDinduced enteropathy was the most common etiology of obscure GI bleeding.

Protein losing enteropathy is another clinical manifestation of NSAID-induced enteropathy, which can lead to hypoalbuminemia. Low serum albumin was detected in $10 \%$ of rheumatoid arthritis patients taking NSAIDs. ${ }^{47}$ In a study using ${ }^{51}$ chromium, the protein loss was observed mainly at the distal ileum level in long-term NSAID users. ${ }^{48}$

Diaphragm-like stricture is a rare, but pathognomonic, feature of NSAID-induced enteropathy. The numerous concentric, luminal projections of fibrotic submucosal tissue may 
cause non-specific or obstructive symptoms. ${ }^{49,50}$ However, because of the intact proper muscle layers, perforation was uncommon. Recently, balloon dilation, instead of surgery, was successfully performed to relieve obstructive symptoms. ${ }^{50}$ In other studies, NSAID-induced enteropathy was reported to be associated with diverticulitis and diverticular bleeding, vitamin $\mathrm{B}_{12}$ deficiency, and the impairment of bile acid absorption. ${ }^{47,51,52}$

\section{DIAGNOSIS}

The most important reason for underestimating the clinical importance of NSAID-induced enteropathy is the difficulty in making a diagnosis. Before introduction of CE and DBE, the diagnosis of NSAID-induced enteropathy relied on the measurement of small bowel permeability and inflammation. To assay the small bowel permeability, orally administered materials should be shown to be rarely absorbed by an intact small bowel barrier, and absorption should occur only in an area of a damaged intestinal barrier. Then, the absorbed materials are excreted in the urine. Therefore, the severity of small bowel injuries could be confirmed through the amount of the excreted materials in the urine. The permeability of chromium-51-labeled EDTA $\left({ }^{51} \mathrm{Cr}\right.$-EDTA $)$ was relatively specific to the small intestine and it was the most widely used in measuring NSAID-induced enteropathy. ${ }^{48,53,54}$ One study using ${ }^{51} \mathrm{Cr}$-EDTA permeability showed that the small bowel was more damaged as the dose of NSAIDs was increased (19\% with $750 \mathrm{mg}$ naproxen vs. $68 \%$ with 1,000 mg naproxen). ${ }^{48}$ The inflammation of the small intestine could also be detected in scintigraphy using ${ }^{111}$ indium (In)-labeled leukocytes. The $50 \%$ to $70 \%$ of patients taking long-term NSAIDs showed enhanced uptake in the small bowel ${ }^{55}$ but this test was difficult to use in clinical practice because of its high cost.

Calprotectin, which is a protein in the cytosol of neutrophils, monocytes, and macrophages, can be used as an inflammatory marker of the small intestine. By checking the amount of fecal calprotectin, small bowel injuries caused by NSAIDs could be estimated. One study showed that the level of a single fecal calprotectin was correlated with the 4-day fecal excretion of ${ }^{111}$ In-labeled leukocytes. ${ }^{53}$ However, fecal calprotectin had low specificity because it could be elevated in IBD, colon cancer, and other inflammatory conditions.

CE and DBE enabled the direct visualization of the small intestine and more exact localization of small bowel injuries. Therefore, the small bowel injuries, caused by NSAIDs, could be better understood using these diagnostic modalities.
Compared with DBE, CE is a painless procedure, and has been used easily to evaluate the small bowel injuries caused by NSAIDs. Hayashi et al. ${ }^{56}$ defined the criteria of NSAIDinduced enteropathy as (1) history of NSAID use; (2) endoscopic findings, such as erosions, ulcers, and diaphragmlike strictures; (3) improvement of clinical manifestations and/or endoscopic findings after stopping the NSAIDs; and (4) exclusion of other etiologies, such as IBD, infection, and malignancy. Various findings such as erosions, ulcers, and strictures were found by CE (Fig. 2). Maiden et al. ${ }^{4}$ divided the NSAID injuries found by CE into 5 categories: petechiae (demarcated areas of crimson mucosa), reddened folds, denuded areas (loss of villi), mucosal breaks (mucosal erosions and/or ulcers), and presence of blood without a visualized lesion. ${ }^{20}$ In this report, mucosal breaks were detected in about $40 \%$ of healthy volunteers after taking $150 \mathrm{mg}$ /day of diclofenac for 2 weeks. In a DBE study, multiple discrete ulcers were found in $28 \%$ of patients taking NSAIDs. ${ }^{13}$ Graham et $\mathrm{al}^{3}{ }^{3}$ reported that mucosal lesions, including red spots, small erosions, large erosions, and ulcers, developed in 13 out of 21 patients (62\%) who were chronic NSAID users. As the ability to find small lesions using CE and DBE was not comprehensive, further advanced modalities should be developed.

\section{PREVENTION AND TREATMENTS}

The most effective method of preventing NSAID-induced enteropathy is to discontinue the NSAIDs. However, even if temporary withdrawal of NSAIDs is possible, it would be medically contraindicated to stop the NSAIDs continuously in patients with chronic pain or anti-platelet therapy. Therefore, prophylactic drugs are essential in chronic NSAID users, especially if there is suspected small bowel bleeding. Until recently, although many efforts have been attempted, there were no methods or medications to prevent or cure NSAID-induced enteropathy. Medications, such as $\mathrm{H}_{2}$ antagonists, sucralfate, or PPIs, which were developed to minimize upper GI injuries caused by NSAIDs, did not prevent the small intestine injuries. ${ }^{10,11}$

COX-2 inhibitors produced less gastroduodenal ulceration and bleeding, compared with non-selective NSAIDs. Therefore, COX-2 inhibitors were thought to be less toxigenic to the small bowel. ${ }^{57,58}$ Goldstein et al. ${ }^{5}$ showed that small bowel injuries were lower in patients taking celecoxib for 2 weeks, compared with naproxen combined with omeprazole. COX2 was thought to be associated with maintenance of the integrity of the tissue, repair of mucosal injury, and resolution 

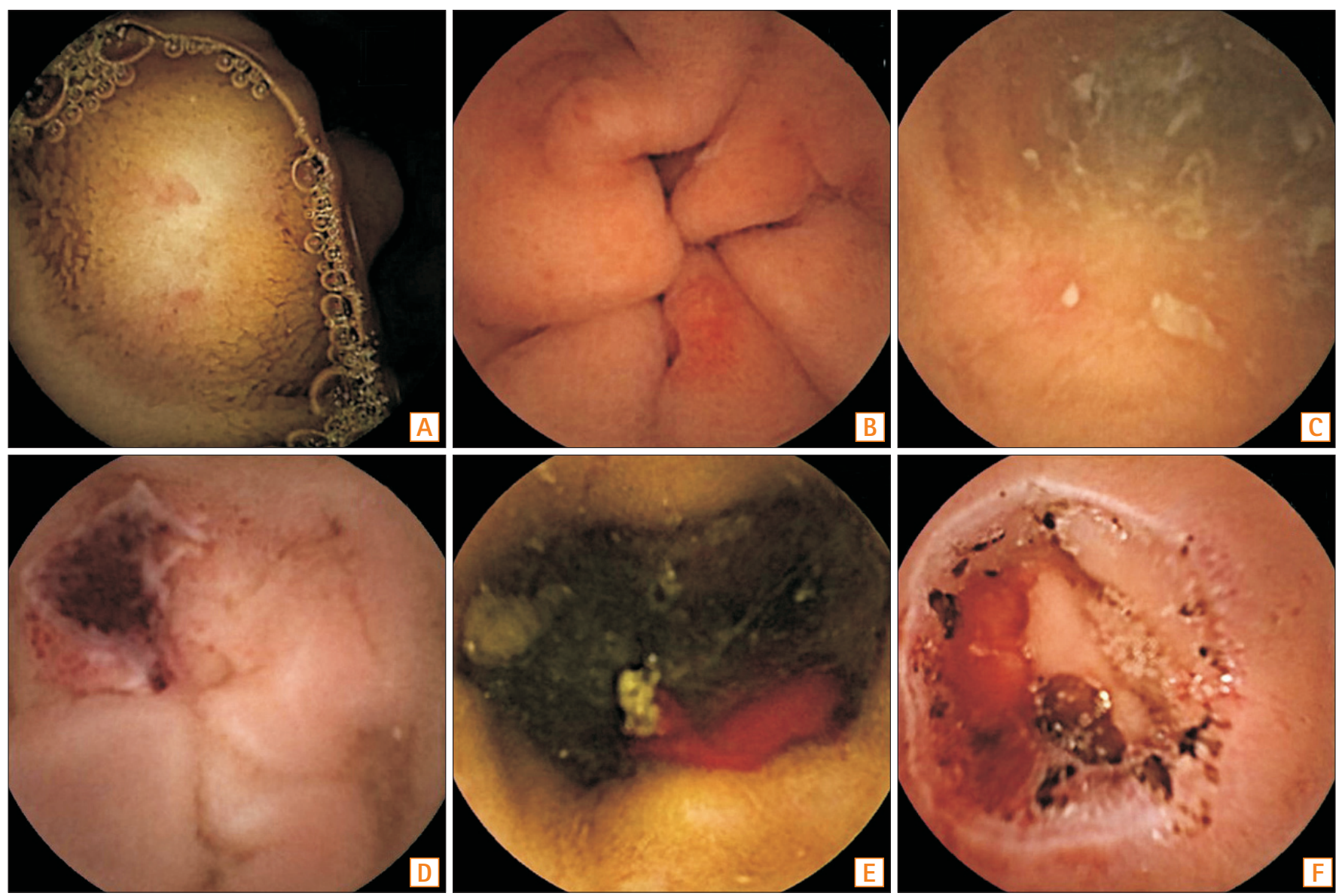

Fig. 2. Capsule endoscopic images of various intestinal mucosal lesions by NSAID. (A) Small bowel petechiae, (B) small bowel erosion, (C) small bowel ulcer, (D) small bowel ulcer with adherent blood clots, (E) small bowel active bleeding, and (F) small bowel stricture with adherent clots.

of inflammation. ${ }^{59,60}$ However, in patients taking COX-2 inhibitors for more than 3 months, there was no significant difference in the incidence of small bowel injuries between the users of selective COX-2 inhibitors and conventional NSAID users. ${ }^{6,1}$ Further studies should be performed to determine whether the COX-2 inhibitors can reduce small bowel toxicities.

PG has an important role in mucosal protection; misoprostol, a synthetic PG analog, demonstrated a decrease in the intestinal permeability caused by NSAIDs in several studies. ${ }^{62,63}$ Watanabe et al. ${ }^{64}$ reported that misoprostol, but not PPI therapy, was effective in improving mucosal injuries using CE in patients who had developed gastric ulcers when taking low-dose aspirin. Conversely, there was no reduction of the intestinal permeability of ${ }^{51} \mathrm{Cr}$-EDTA in patients taking indomethacin, even when low dose of misoprostol was given. ${ }^{47}$ Despite the effectiveness in treating NSAID-induced enteropathy, misoprostol causes many GI adverse effects, such as nausea, indigestion, abdominal pain, and diarrhea. ${ }^{65}$ These side effects could limit its clinical use.

Rebamipide is a drug that promotes GI mucosal protection by increasing mucus and stimulating PG synthesis. ${ }^{66,67}$ It also scavenges free radicals and suppresses myeloperoxidase activity. In a study using CE, Niwa et al. ${ }^{68}$ showed its effectiveness in treating NSAID-induced enteropathy in healthy humans. Small intestine injuries were less frequent in the patients receiving diclofenac for 7 days, together with rebamipide, than in those receiving a placebo $(20 \%$ with rebamipide vs. $80 \%$ with placebo). However, in the larger study, there was no difference between the group taking diclofenac, PPI, and rebamipide for 14 days and the group taking diclofenac, PPI, and a placebo. ${ }^{69}$ Furthermore, additional studies should be performed to clarify the effectiveness of rebamipide in treating NSAID-induced enteropathy.

Metronidazole is an antibiotic, which is effective against many enteric anaerobic bacteria. Bjarnason et al. ${ }^{70}$ reported 
that co-administered metronidazole (with NSAIDs) was effective in reducing NSAID-induced enteropathy. The patients taking NSAIDs received metronidazole and the fecal excretion of ${ }^{51} \mathrm{Cr}$-labeled erythrocytes and ${ }^{111}$ In-labeled neutrophils was measured. These 2 fecal inflammatory markers were reduced with the treatment of metronidazole. Recently, several studies have evaluated the potential value of probiotics for prevention or treatment of NSAID-induced enteropathy. ${ }^{45,71,72}$ In animal models, Lactobacillus acidophilus and Bifidobacterium adolescentis reduced ileal ulcer formation in rats treated with NSAIDs. ${ }^{70}$ In a clinical trial, CE showed a significant reduction of mucosal breaks in the Lactobacillus casei group compared with the placebo group in patients receiving low-dose enteric-coated aspirin and omeprazole treatment for more than 3 months. ${ }^{45}$ Additionally, VSL\#3, a probiotic formulation consisting of 8 different species of microorganisms, was effective in reducing the fecal calprotectin level in volunteers taking indomethacin. ${ }^{72}$

PPIs strongly inhibit gastric acid secretion and are prescribed for prevention and treatment of gastroduodenal ulcers by NSAIDs. A previous study found that lansoprazole was effective in reducing small bowel injuries caused by NSAIDs in animal models. ${ }^{73}$ The mechanism of preventing small bowel injuries by lansoprazole was thought to be by inducing the heme oxygenase-1, which has an important role in inhibiting NSAID-induced small bowel injuries. ${ }^{73,74}$ Pretreatment with tin-protoporphyrin, which is an inhibitor of heme oxygenase-1, increased the indomethacin-induced enteropathy. On the contrary, lansoprazole exacerbated the small bowel injuries in rats treated with naproxen. ${ }^{75} \mathrm{In}$ a clinical study using CE, small bowel injuries were found more commonly in patients treated with naproxen and omeprazole (55\%) than those treated with other medications ( $16 \%$ with celecoxib only, $7 \%$ in controls). ${ }^{5}$ The aggravation of NSAID-induced small bowel injuries by PPIs could be explained by their potential to cause a shift in the types of bacteria in the small intestine (dysbiosis). ${ }^{44}$ The rats treated with omeprazole had a significant reduction in Actinobacteria, particularly Bifidobacteria, in the jejunum; however, the addition of Bifidobacteria in PPI-treated rats reduced the NSAID-induced intestinal damage. ${ }^{44}$ For lansoprazole, a clinical trial will be needed to confirm the effect, considering the 2 conflicting reports.

Several drugs, such as NO-releasing NSAIDs and hydrogen sulfide $\left(\mathrm{H}_{2} \mathrm{~S}\right)$-releasing NSAIDs have been developed using the co-drug model. The $\mathrm{NO}$ or $\mathrm{H}_{2} \mathrm{~S}$ portions of the co-drugs promote mucosal protection via increasing mucosal blood flow and inhibiting leukocyte adherence to the endothelium.
In a clinical trial, NO-naproxen decreased small bowel permeability compared with an equivalent dose of naproxen. ${ }^{75}$ Additionally, $\mathrm{H}_{2} \mathrm{~S}$-releasing NSAIDs showed enhanced antiinflammatory activity in comparison to the conventional NSAIDs. ${ }^{76-78}$

Phospholipid has been proposed to reduce the topical irritant property of NSAIDs. ${ }^{79-81}$ In those studies, it was a component of the epithelial barrier to acid-back diffusion and had an important role in preventing the NSAIDs from disrupting the barrier. The phospholipid lining of the mucosal surface, which was hydrophobic, suppressed the invasion of acid, bile, and other toxic materials. In an animal study, small bowel injuries were not found in the phosphatidylcholine (PC)-indomethacin group. ${ }^{82}$ In clinical trials, PC-ibuprofen reduced the gastroduodenal injuries, as compared to ibuprofen. ${ }^{83}$ However, there are no clinical studies regarding the effectiveness of PC-NSAIDs in small bowel injuries.

Sulfasalazine may be a possible treatment modality in NSAID-induced enteropathy. In rheumatoid arthritis patients taking NSAIDs, sulfasalazine reduced intestinal inflammation and blood loss, whereas disease-modifying antirheumatic drugs did not. ${ }^{84}$

\section{CONCLUSIONS}

NSAID-induced enteropathy may be as frequent and severe as upper GI complications. In most cases, clinical manifestations are non-specific and pathogenic mechanisms are not well known, but are suspected to be complicated. The new diagnostic modalities, such as CE and DBE, enable diagnosis of small bowel injuries caused by NSAIDs more easily than in the past. However, there is no proven effective medication for treating NSAID-induced enteropathy. Therefore, further studies regarding the prevention and treatment of intestinal injuries caused by NSAIDs are urgently needed.

\section{REFERENCES}

1. Fries JF, Williams CA, Bloch DA, Michel BA. Non-steroidal antiinflammatory drug-associated gastropathy: incidence and risk factor models. Am J Med 1991;91:213-222.

2. Smalley WE, Ray WA, Daugherty JR, Griffin MR. Nonsteroidal anti-inflammatory drugs and the incidence of hospitalizations for peptic ulcer disease in elderly persons. Am J Epidemiol 1995;141:539-545.

3. Graham DY, Opekun AR, Willingham FF, Qureshi WA. Visible small-intestinal mucosal injury in chronic NSAID users. Clin Gastroenterol Hepatol 2005;3:55-59. 
4. Maiden L, Thjodleifsson B, Theodors A, Gonzalez J, Bjarnason I. A quantitative analysis of NSAID-induced small bowel pathology by capsule enteroscopy. Gastroenterology 2005;128:11721178.

5. Goldstein JL, Eisen GM, Lewis B, et al. Video capsule endoscopy to prospectively assess small bowel injury with celecoxib, naproxen plus omeprazole, and placebo. Clin Gastroenterol Hepatol 2005;3:133-141.

6. Maiden L, Thjodleifsson B, Seigal A, et al. Long-term effects of nonsteroidal anti-inflammatory drugs and cyclooxygenase-2 selective agents on the small bowel: a cross-sectional capsule enteroscopy study. Clin Gastroenterol Hepatol 2007;5:10401045.

7. Maiden L. Capsule endoscopic diagnosis of nonsteroidal antiinflammatory drug-induced enteropathy. J Gastroenterol 2009;44 Suppl 19:64-71.

8. Lanas A, García-Rodríguez LA, Polo-Tomás M, et al. Time trends and impact of upper and lower gastrointestinal bleeding and perforation in clinical practice. Am J Gastroenterol 2009;104:1633-1641.

9. Hudson N, Hawkey CJ. Non-steroidal anti-inflammatory drugassociated upper gastrointestinal ulceration and complications. Eur J Gastroenterol Hepatol 1993;5:412-419.

10. Park SC, Chun HJ, Kang CD, Sul D. Prevention and management of non-steroidal anti-inflammatory drugs-induced small intestinal injury. World J Gastroenterol 2011;17:4647-4653.

11. Lim YJ, Yang CH. Non-steroidal anti-inflammatory druginduced enteropathy. Clin Endosc 2012;45:138-144.

12. Allison MC, Howatson AG, Torrance CJ, Lee FD, Russell RI. Gastrointestinal damage associated with the use of nonsteroidal anti-inflammatory drugs. N Engl J Med 1992;327:749-754.

13. Matsumoto T, Kudo T, Esaki M, et al. Prevalence of non-steroidal anti-inflammatory drug-induced enteropathy determined by double-balloon endoscopy: a Japanese multicenter study. Scand J Gastroenterol 2008;43:490-496.

14. Endo H, Hosono K, Inamori M, et al. Characteristics of small bowel injury in symptomatic chronic low-dose aspirin users: the experience of two medical centers in capsule endoscopy. J Gastroenterol 2009;44:544-549.

15. Leung WK, Bjarnason I, Wong VW, Sung JJ, Chan FK. Small bowel enteropathy associated with chronic low-dose aspirin therapy. Lancet 2007;369:614.

16. Stattery J, Warlow CP, Shorrock CJ, Langman MJ. Risks of gastrointestinal bleeding during secondary prevention of vascular events with aspirin: analysis of gastrointestinal bleeding during the UK-TIA trial. Gut 1995;37:509-511.
17. Bjarnason I, Hayllar J, MacPherson AJ, Russell AS. Side effects of nonsteroidal anti-inflammatory drugs on the small and large intestine in humans. Gastroenterology 1993;104:1832-1847.

18. Wallace JL. NSAID gastropathy and enteropathy: distinct pathogenesis likely necessitates distinct prevention strategies. $\mathrm{Br} \mathrm{J}$ Pharmacol 2012;165:67-74.

19. Robert A, Asano T. Resistance of germ-free rats to indomethacin induced intestinal inflammation. Prostaglandins 1977;14:333341.

20. Fang WF, Broughton A, Jacobson ED. Indomethacin induced intestinal inflammation. Am J Dig Dis 1977;22:749-760.

21. Whittle BJ. Temporal relationship between cyclooxygenase inhibition, as measured by prostacyclin biosynthesis, and the gastrointestinal damage induced by indomethacin in the rat. Gastroenterology 1981;80:94-98.

22. Yamada T, Deitch E, Specian RD, Perry MA, Sartor RB, Grisham MB. Mechanisms of acute and chronic intestinal inflammation induced by indomethacin. Inflammation 1993;17:641-662.

23. Sigthorsson G, Simpson RJ, Walley M, et al. COX-1 and 2, intestinal integrity, and pathogenesis of nonsteroidal antiinflammatory drug enteropathy in mice. Gastroenterology 2002;122:1913-1923.

24. Wax J, Clinger WA, Varner P, Bass P, Winder CV. Relationship of the enterohepatic cycle to ulcerogenesis in the rat small bowel with flufenamic acid. Gastroenterology 1970;58:772-780.

25. Jacob M, Foster R, Sigthorsson G, Simpson R, Bjarnason I. Role of bile in pathogenesis of indomethacin-induced enteropathy. Arch Toxicol 2007;81:291-298.

26. Lichtenberger LM, Phan T, Okabe S. Aspirin's ability to induce intestinal injury in rats is dependent on bile and can be reversed if pre-associated with phosphatidylcholine. J Physiol Pharmacol 2011;62:491-496.

27. Dammann HG, Burkhardt F, Wolf N. Enteric coating of aspirin significantly decreases gastroduodenal mucosal lesions. Aliment Pharmacol Ther 1999;13:1109-1114.

28. Blondon H, Barbier JP, Mahé I, Deverly A, Kolsky H, Bergmann JF. Gastroduodenal tolerability of medium dose enteric-coated aspirin: a placebo controlled endoscopic study of a new enteric coated formation versus regular formation in healthy volunteers. Fundam Clin Pharmacol 2000;14:155-157.

29. Lanza FL, Royer GL Jr, Nelson RS. Endoscopic evaluation of the effects of aspirin, buffered aspirin, and enteric-coated aspirin on gastric and duodenal mucosa. N Engl J Med 1980;303:136138.

30. Petroski D. Endoscopic comparison of three aspirin preparations and placebo. Clin Ther 1993;15:314-320. 
31. Reuter BK, Davies NM, Wallace JL. Nonsteroidal anti-inflammatory drug enteropathy in rats: role of permeability, bacteria, and enterohepatic circulation. Gastroenterology 1997;112:109-117.

32. Somasundaram S, Rafi S, Hayllar J, et al. Mitochondrial damage: a possible mechanism of the "topical" phase of NSAID induced injury to the rat intestine. Gut 1997;41:344-353.

33. Uejima M, Kinouchi T, Kataoka K, Hiraoka I, Ohnishi Y. Role of intestinal bacteria in ileal ulcer formation in rats treated with a nonsteroidal antiinflammatory drug. Microbiol Immunol 1996;40:553-560.

34. Watanabe T, Higuchi K, Kobata A, et al. Non-steroidal antiinflammatory drug-induced small intestinal damage is Toll like receptor 4 dependent. Gut 2008;57:181-187.

35. Koga H, Aoyagi K, Matsumoto T, Iida M, Fujishima M. Experimental enteropathy in athymic and euthymic rats: synergistic role of lipopolysaccharide and indomethacin. Am J Physiol 1999;276(3 Pt 1):G576-G582.

36. Whittle BJ. Nitric oxide and the gut injury induced by nonsteroidal anti-inflammatory drugs. Inflammopharmacology 2003;11:415-422.

37. Santucci L, Fiorucci S, Di Matteo FM, Morelli A. Role of tumor necrosis factor alpha release and leukocyte margination in indomethacin-induced gastric injury in rats. Gastroenterology 1995;108:393-401.

38. Reuter BK, Wallace JL. Phosphodiesterase inhibitors prevent NSAID enteropathy independently of effects on TNF alpha release. Am J Physiol 1999;277(4 Pt 1):G847-G854.

39. Bertrand V, Guimbaud R, Tulliez M, et al. Increase in tumor necrosis factor-alpha production linked to the toxicity of indomethacin for the rat small intestine. Br J Pharmacol 1998;124:1385-1394.

40. LoGuidice A, Wallace BD, Bendel L, Redinbo MR, Boelsterli UA. Pharmacologic targeting of bacterial beta-glucuronidase alleviates nonsteroidal anti-inflammatory drug-induced enteropathy in mice. J Pharmacol Exp Ther 2012;341:447-454.

41. Lombardo L, Foti M, Ruggia O, Chiecchio A. Increased incidence of small intestinal bacterial overgrowth during proton pump inhibitor therapy. Clin Gastroenterol Hepatol 2010;8:504508.

42. Compare D, Pica L, Rocco A, et al. Effects of long-term PPI treatment on producing bowel symptoms and SIBO. Eur J Clin Invest 2011;41:380-386.

43. Jacobs C, Coss Adame E, Attaluri A, Valestin J, Rao SS. Dysmotility and proton pump inhibitor use are independent risk factors for small intestinal bacterial and/or fungal overgrowth. Aliment Pharmacol Ther 2013;37:1103-1111.
44. Wallace JL, Syer S, Denou E, et al. Proton pump inhibitors exacerbate NSAID-induced small intestinal injury by inducing dysbiosis. Gastroenterology 2011;141:1314-1322.

45. Endo H, Higurashi T, Hosono K, et al. Efficacy of Lactobacillus casei treatment on small bowel injury in chronic low-dose aspirin users: a pilot randomized controlled study. J Gastroenterol 2011;46:894-905.

46. Kameda N, Higuchi K, Shiba M, et al. A prospective, singleblind trial comparing wireless capsule endoscopy and doubleballoon enteroscopy in patients with obscure gastrointestinal bleeding. J Gastroenterol 2008;43:434-440.

47. Davies NM, Saleh JY, Skjodt NM. Detection and prevention of NSAID-induced enteropathy. J Pharm Pharm Sci 2000;3:137155.

48. Aabakken L, Osnes M. 51Cr-ethylenediaminetetraacetic acid absorption test: effects of naproxen, a non-steroidal, antiinflammatory drug. Scand J Gastroenterol 1990;25:917-924.

49. Santolaria S, Cabezali R, Ortego J, et al. Diaphragm disease of the small bowel: a case without apparent nonsteroidal antiinflammatory drug use. J Clin Gastroenterol 2001;32:344-346.

50. Sunada K, Yamamoto H. Double-balloon endoscopy: past, present, and future. J Gastroenterol 2009;44:1-12.

51. Rahme E, Barkun A, Nedjar H, Gaugris S, Watson D. Hospitalizations for upper and lower GI events associated with traditional NSAIDs and acetaminophen among the elderly in Quebec, Canada. Am J Gastroenterol 2008;103:872-882.

52. Zuccaro G. Epidemiology of lower gastrointestinal bleeding. Best Pract Res Clin Gastroenterol 2008;22:225-232.

53. Lanas A, Sopeña F. Nonsteroidal anti-inflammatory drugs and lower gastrointestinal complications. Gastroenterol Clin North Am 2009;38:333-352.

54. Davies GR, Rampton DS. The pro-drug sulindac may reduce the risk of intestinal damage associated with the use of conventional non-steroidal anti-inflammatory drugs. Aliment Pharmacol Ther 1991;5:593-598.

55. Davies NM. Sustained release and enteric coated NSAIDs: are they really GI safe? J Pharm Pharm Sci 1999;2:5-14.

56. Hayashi Y, Yamamoto H, Kita H, et al. Non-steroidal anti-inflammatory drug-induced small bowel injuries identified by doubleballoon endoscopy. World J Gastroenterol 2005;11:4861-4864.

57. Smecuol E, Bai JC, Sugai E, et al. Acute gastrointestinal permeability responses to different non-steroidal anti-inflammatory drugs. Gut 2001;49:650-655.

58. Takeuchi K, Tanaka A, Kato S, Amagase K, Satoh H. Roles of COX inhibition in pathogenesis of NSAID-induced small intestinal damage. Clin Chim Acta 2010;411:459-466. 
59. Wallace JL, Devchand PR. Emerging roles for cyclooxygenase-2 in gastrointestinal mucosal defense. Br J Pharmacol 2005;145:275-282.

60. Sugimori S, Watanabe T, Tabuchi M, et al. Evaluation of small bowel injury in patients with rheumatoid arthritis by capsule endoscopy: effects of anti-rheumatoid arthritis drugs. Digestion 2008;78:208-213.

61. Laine L, Curtis SP, Langman M, et al. Lower gastrointestinal events in a double-blind trial of the cyclo-oxygenase-2 selective inhibitor etoricoxib and the traditional nonsteroidal anti-inflammatory drug diclofenac. Gastroenterology 2008;135:15171525.

62. Fujimori S, Seo T, Gudis K, et al. Prevention of nonsteroidal anti-inflammatory drug-induced small-intestinal injury by prostaglandin: a pilot randomized controlled trial evaluated by capsule endoscopy. Gastrointest Endosc 2009;69:1339-1346.

63. Fujimori S, Takahashi Y, Seo T, et al. Prevention of traditional NSAID-induced small intestinal injury: recent preliminary studies using capsule endoscopy. Digestion 2010;82:167-172.

64. Watanabe T, Sugimori S, Kameda N, et al. Small bowel injury by low dose enteric-coated aspirin and treatment with misoprostol: a pilot study. Clin Gastroenterol Hepatol 2008;6:1279-1282.

65. Fortun PJ, Hawkey CJ. Nonsteroidal antiinflammatory drugs and the small intestine. Curr Opin Gastroenterol 2007;23:134141.

66. Arakawa T, Watanabe T, Fukuda T, Yamasaki K, Kobayashi K. Rebamipide, novel prostaglandin-inducer accelerates healing and reduces relapse of acetic acid-induced rat gastric ulcer: comparison with cimetidine. Dig Dis Sci 1995;40:2469-2472.

67. Higuchi K, Umegaki E, Watanabe T, et al. Present status and strategy of NSAIDs-induced small bowel injury. J Gastroenterol 2009;44:879-888.

68. Niwa Y, Nakamura M, Ohmiya N, et al. Efficacy of rebamipide for diclofenac induced small-intestinal mucosal injuries in healthy subjects: a prospective, randomized, double-blinded, placebo controlled, cross-over study. J Gastroenterol 2008;43:270-276.

69. Fujimori S, Takahashi Y, Gudis K, et al. Rebamipide has the potential to reduce the intensity of NSAID-induced small intestinal injury: a double blind, randomized, controlled trial evaluated by capsule endoscopy. J Gastroenterol 2011;46:57-64.

70. Bjarnason I, Hayllar J, Smethurst P, Price A, Gumpel MJ. Metronidazole reduces intestinal inflammation and blood loss in non-steroidal anti-inflammatory drug induced enteropathy. Gut 1992;33:1204-1208.
71. Kinouchi T, Kataoka K, Bing SR, et al. Culture supernatants of Lactobacillus acidophilus and Bifidobacterium adolescentis repress ileal ulcer formation in rats treated with a nonsteroidal anti-inflammatory drug by suppressing unbalanced growth of aerobic bacteria and lipid peroxidation. Microbiol Immunol 1998;42:347-355.

72. Montalto M, Gallo A, Curigliano V, et al. Clinical trial: the effects of a probiotic mixture on non-steroidal anti-inflammatory drug enteropathy: a randomized, double blind, cross-over, placebocontrolled study. Aliment Pharmacol Ther 2010;32:209-214.

73. Yoda Y, Amagase K, Kato S, et al. Prevention by lansoprazole, a proton pump inhibitor, of indomethacin-induced small intestinal ulceration in rats through induction of heme oxygenase-1. J Physiol Pharmacol 2010;61:287-294.

74. Higuchi K, Yoda Y, Amagase K, et al. Prevention of NSAIDinduced small bowel intestinal mucosal injury: prophylactic potential of lansoprazole. J Clin Biochem Nutr 2009;45:125-130.

75. Hawkey CJ, Jones JI, Atherton CT, et al. Gastrointestinal safety of AZD3582, a cyclooxygenase inhibiting nitric oxide donator: proof of concept study in humans. Gut 2003;52:1537-1542.

76. Sparatore A, Perrino E, Tazzari V, et al. Pharmacological profile of a novel H(2)S-releasing aspirin. Free Radic Biol Med 2009;46:586-592.

77. Wallace JL, Vong L, McKnight W, Dicay M, Martin GR. Endogenous and exogenous hydrogen sulfide promotes resolution of colitis in rats. Gastroenterology 2009;137:569-578.

78. Wallace JL, Ferraz JG, Muscara MN. Hydrogen sulfide: an endogenous mediator of resolution of inflammation and injury. Antioxid Redox Signal 2012;17:58-67.

79. Lichtenberger LM, Wang ZM, Romero JJ, et al. Non-steroidal anti-inflammatory drugs (NSAIDs) associate with zwitterionic phospholipids: insight into the mechanism and reversal of NSAID-induced gastrointestinal injury. Nat Med 1995;1:154158.

80. Lichtenberger LM, Zhou Y, Dial EJ, Raphael RM. NSAID injury to the gastrointestinal tract: evidence that NSAIDs interact with phospholipids to weaken the hydrophobic surface barrier and induce the formation of unstable pores in membranes. J Pharm Pharmacol 2006;58:1421-1428.

81. Lichtenberger LM, Barron M, Marathi U. Association of phosphatidylcholine and NSAIDs as a novel strategy to reduce gastrointestinal toxicity. Drugs Today (Barc) 2009;45:877-890.

82. Lim YJ, Phan TM, Dial EJ, Graham DY, Lichtenberger LM. In vitro and in vivo protection against indomethacin induced small intestinal injury by proton pump inhibitors, acid pump antagonists, or indomethacin-phosphatidylcholine. Digestion 2012;86:171-177. 
83. Lanza FL, Marathi UK, Anand BS, Lichtenberger LM. Clinical trial: comparison of ibuprofen-phosphatidylcholine and ibuprofen on the gastrointestinal safety and analgesic efficacy in osteoarthritic patients. Aliment Pharmacol Ther 2008;28:431442.
84. Hayllar J, Smith T, Macpherson A, Price AB, Gumpel M, Bjarnason I. Nonsteroidal anti-inflammatory drug-induced small intestinal inflammation and blood loss: effects of sulfasalazine and other disease-modifying antirheumatic drugs. Arthritis Rheum 1994;37:1146-1150. 